\title{
Sleeping with Tuberculosis: Do over-crowded university hostels in Kenya increase TB transmission among youths - A pilot study
}

\section{Maina Teresia}

Department of Public Health, Pwani University, Kilifi, Kenya

\section{Willets Annie}

Queen Margret University, Edinburgh, United Kingdom

\section{Ngari Moses}

KEMRI Wellcome Trust Research Programme, Kilifi, Kenya

Osman Abdullahi ( $\nabla$ o.abdullahi@pu.ac.ke)

Pwani University https://orcid.org/0000-0001-5003-2973

\section{Research article}

Keywords: Contact investigation, Students, Index cases, Clinical TB, GeneXpert

Posted Date: May 28th, 2020

DOI: https://doi.org/10.21203/rs.3.rs-30747/v1

License: (a) (i) This work is licensed under a Creative Commons Attribution 4.0 International License. Read Full License 


\section{Abstract \\ Background}

Understanding the magnitude of Tuberculosis (TB) transmission among the youth is a global priority as the disease burden shifts to this population. Learning institutions host overcrowded accommodation and classrooms, especially in resource limited contexts. Understanding the global threat of the youth as an infection pool on the wider population is highlighted in the global response to COVID 19. This pilot study aimed to test the feasibility of recruiting university students' contacts and demonstrate transmission of TB in Pwani University, Kilifı County-Kenya.

\section{Materials and Methods}

A pilot study among Pwani University TB index cases receiving treatment at the Kilifi County Hospital was conducted. Index cases who consented provided information about their household and social contacts. Contacts were identified and screened using a World Health Organization (WHO) symptom-based questionnaire. Their sputum samples were analysed using GeneXpert. Multivariate log-binomial regression was used to determine demographic and clinical characteristics associated with TB infection among contacts with TB index patients.

\section{Results}

A total of 51 index cases were recruited, median (IQR) age of 21 (20-23) years and 31 (61\%) were males. 156 contacts were screened, median (IQR) age of $23(20-23)$ years, $80(51 \%)$ were males and $76(49 \%)$ were household contacts. Among the 156 TB contacts, 5 participants were confirmed positive for TB: prevalence of $3.2 \%$ (95\% Cl 1.0 to $7.3 \%) .8 / 156$ (5.1\%, (95\% Cl 2.2 to $10 \%)$ contacts, had clinical diagnosed TB despite having a negative GeneXpert result. In total 13/156 contacts had either confirmed or clinical diagnosed $\mathrm{TB} ; 8.3 \%$ (95\% $\mathrm{Cl} 4.5$ to $14 \%)$. Sharing a bed with an index case was the only factor significantly associated with TB infection among the five contacts with GeneXpert diagnosed TB.

\section{Conclusion}

Students sleeping in crowded hostels promote TB transmission within universities informing TB control interventions. Collaborating with existing national TB programme systems is a feasible approach to recruit people with active disease and their social contacts. Expansion of this approach to a larger population of students with TB infection may demonstrate the magnitude of TB transmission within universities, and the wider local communities.

\section{Background}


Tuberculosis (TB) has existed for decades and remains a top global health problem [1]. Globally, in 2017, 10 million people became ill and 1.6 million succumbed to the disease [2]. TB affects all age groups; although, recently, the burden of the disease has shifted to young adults who form economically productive people in society. A global estimate of TB prevalence among young adults aged 15-24 years in 2012 revealed that 1.78 million youths had contracted TB [3].

There is limited data on the barriers to TB diagnosis and treatment in the youth population. The few studies conducted report that barriers to testing and treatment are attributed to stigma, perception of TB, knowledge about the illness, availability of diagnostic tests as well as access to care [4-6]. These TB control challenges further compound the TB burden among the youth .

Contact investigation in high-income countries with low disease burden aims to rapidly identify persons with active and latent TB infection [7]. Studies reveal that tracing among household contacts identifies more TB cases than passive case finding approach [8, 9]. A systematic review and meta-analysis that involved 95 contact investigation studies from resource limited countries showed a $3.1 \%$ prevalence of TB among household contacts with the highest incidence occurring in the first 12 months after exposure [10]. In resource-limited settings, contact investigation is included in the national TB control and prevention programmes, however is inconsistently and rarely performed due to lack of clear definitions of indexes, contacts, and procedures.

Studies conducted in congregate settings found overcrowding, poor ventilation, psychosocial factors, and close contact with TB patients for extended duration [10-12] contribute to TB transmission among high risk groups. A high burden of TB has been observed among the student population for instance, a study conducted at Addis Ababa University, Sidist Kilo campus and the Adama Science and Technology University reported a prevalence of $511.7 / 100,000$ and $1098.1 / 100,000$ respectively [14]. In tertiary learning institutions, one case of TB can have numerous close contacts which could result in increased cases of active disease or latent infections [13].

Kenya is among the 30 countries with a high TB disease burden [1]. The recent prevalence of pulmonary TB in Kenya for people above the age of 15 years was 558 per 100,000 [15]. There is limited data on TB transmission among university students in Kenya. The aim of this pilot study was to test the feasibility of recruiting university students' contacts and demonstrate transmission of TB in Pwani University, Kilifi County-Kenya.

\section{Methods}

\section{Study design}

A pilot study was conducted among people attending the university in contact with students with active TB disease in Kilifi, Kenya from January 2016 to December 2017.

\section{Study participants}


All Pwani university students diagnosed with TB at Kilifi County Referral Hospital (KCH) during the study period and consented to participate were recruited as index cases. The index case was a university student who tested positive for TB and treated at KCH in the years 2016 and 2017, and resided in the university hostels or off-campus. Students diagnosed with TB were identified from the hospital register and online TB patient surveillance database (TIBU). After consenting to participate in the study, index participants provided information about their household and social contacts. A household contact was defined as a person who shared an enclosed living (hostel) with an index case for one or more nights or for frequent periods during three months before the commencement of TB treatment. A social contact was defined as a person not in the same hostel as an index case but shared enclosed space such as lecture hall, dining area, or library for extended periods during the day in the last three months before the commencement of TB treatment.

\section{Study Setting}

This study was conducted at Pwani University with a student population of 8,600 in Kilifi Town. All Pwani university students residing in hostels within the campus and off-campus were eligible to participate in this study. The university hostels are fitted with bunk beds that accommodate two to four students per room. Due to limited accommodation within campus about $90 \%$ of the students reside off-campus in hostels within a three-kilometre radius from the university. This prompts students to share facilities, including beds, in the small rooms.

The university has a health facility on campus however; students suspected to have TB are referred to $\mathrm{KCH}$, a secondary referral hospital located within a two kilometre radius of the university.

All students receiving treatment for TB at KCH in January 2016 and December 2017 were requested for their consent to participate after receiving a detailed explanation of the purpose, benefit and risk of participation in this study.

This study took place in collaboration with personnel at $\mathrm{KCH}$ including a sub-county coordinator, community health volunteers (CHV) and a laboratory technologist. The sub-county coordinator provided access to TIBU, an electronic surveillance system registering all TB patients within the first month of commencing treatment [16]. The TIBU system was used in conjunction with the hospital paper-based register to identify index cases. The sub-county coordinator informed the investigator of newly diagnosed TB cases during the study. The Pwani University Health facility was contacted to validate index and contact data.

The WHO standardized symptom-based questionnaire applied to interview and collect demographic and clinical data from contacts of consenting TB patients [1]. All identified social and household contacts of a student receiving TB treatment comunicated through text messages and mobile phone calls. These participants were contacted up to three times where no response was obtained in the first attempt. Failure to attend the third meeting resulted in exclusion from the study. Contacts were screened for TB signs and 
symptoms (cough, fever, night sweats, swelling/pain on different sites). They were then provided with a falcon tube and asked to produce an early morning sputum sample for the GeneXpert test.

All specimens collected from a patient suspected to have TB were checked for quality and registered in the laboratory logbook. In the laboratory, the standard procedure for sample processing was as follows; the cartridge containing the mixture was placed in the GeneXpert machine which processed the specimen automatically. Results of the GeneXpert Mycobacterium tuberculosis complex (MTBC) were then detected in the sample. In case MTBC was detected, the results also stated whether resistance to the standard TB treatment Rifampicin was detected or not.

\section{Quality Control}

\section{Training}

The researcher attended a TB training workshop to develop skills and a supportive network of county and community-based health providers to support this active contact tracing and TB screening process. The training ensured quality control in each step of the process including recruitment of index patients and contacts, follow up in the community for students living off-campus, and sample collection.

\section{Sample processing}

Each test included a Sample Processing Control (SPC) and a probe check (PCC). SPC ensured the samples were correctly processed. The SPC contains non-infectious spores in the form of a dry spore cake that is included in each cartridge to verify the adequate processing of Mycobacterium tuberculosis (MTB). The SPC verifies the lysis of MTB that has occurred if the organisms are present and whether specimen processing is adequate. Additionally, this control detects specimen associated inhibition of the real-time PCR assay. The SPC should be positive in a negative sample and can be negative or positive in a positive sample. The SPC passes if it meets the validated acceptance criteria. The test result is "Invalid" if the SPC is not detected in a negative test.

\section{Ethical considerations}

Ethical approval was obtained from Pwani University Ethical Review Board (ERC/MSc/ 002/2017). Permission to obtain information about the students with TB infection was obtained from the Kilifi County Ethical Research Committee.

\section{Statistical methods}

Data were recorded on standardized questionnaires and register books and later later coded and entered into the Epinfo database. Statistical analyses were performed using statistical package for social sciences (SPSS) version 25 and R statistical software version 3.4.1 for windows. Descriptive summaries were used to describe TB patients' and contacts' demographic and clinical characteristics. A difference in participants' features between contacts who contracted TB and who did not was tested using chisquare/fisher's exact test as appropriate. Because this method only tests for difference and not the effect 
of individual feature, a sensitivity analysis using a log-binomial regression model albeit with low statistical power was performed. Univariate log-binomial regression for all the features was performed and a backward stepwise approach to select features to retain in the multivariate regression model reporting only features with a $\mathrm{P}<0.05$ done. For the regression analysis, risk ratio (RR) with $95 \%$ confidence intervals $(\mathrm{Cl})$ were reported. In this pilot study, the number of index cases was fixed, therefore no formal sample size was estimated. An exploratory approach was adopted where we did not limit the number of contacts each index case could make.

\section{Results}

\section{Characteristics of Index Students}

From January 2016 to December 2017, a total of 65 students were identified as index TB cases on treatment from $\mathrm{KCH}$. Of these 65 index TB cases, $6(9.2 \%)$ refused to consent and $8(12 \%)$ were not within the area of study, we, therefore, included 51 (79\%) in the study (Fig. 1). Their median (IQR) age was 21 $(20-23)$ years, and $31(61 \%)$ were males. A total of $43(84 \%)$ of these 51 index cases were off-campus residents, 50 (98\%) were new TB cases and 47 (92\%) were PTB patients. GeneXpert was used to diagnose 22 (43\%) of the TB index cases. Only 1 (1.9\%) TB patient was HIV infected while 42 (82\%) of the TB index cases were self-referrals. A total of $10(20 \%)$ of the index TB cases had completed treatment however, we could only confirm $38(74 \%)$ as having been cured (Table 1$)$. 
Table 1

Demographic and clinical characteristics of TB index cases

\begin{tabular}{|c|c|c|}
\hline & $N(51)$ & $(\%)$ \\
\hline \multicolumn{3}{|l|}{ Demographic characteristics } \\
\hline Age, median (IQR) years & \multicolumn{2}{|c|}{$21(20-23)$} \\
\hline \multicolumn{3}{|l|}{ Gender } \\
\hline Male & 31 & 61 \\
\hline Female & 20 & 39 \\
\hline \multicolumn{3}{|l|}{ Residence } \\
\hline In-Campus (IC) & 8 & 16 \\
\hline Off-Campus (OFC) & 43 & 84 \\
\hline \multicolumn{3}{|l|}{ Clinical characteristics } \\
\hline \multicolumn{3}{|l|}{ Type of patient } \\
\hline New & 50 & 98 \\
\hline $\mathrm{TFL}^{\#}$ & 1 & 1.9 \\
\hline \multicolumn{3}{|l|}{ Type of TB } \\
\hline EPTB $^{\$}$ & 4 & 7.8 \\
\hline PTB $\%$ & 47 & 92 \\
\hline \multicolumn{3}{|l|}{ Method of diagnosis } \\
\hline X-ray & 29 & 57 \\
\hline GeneXpert & 22 & 43 \\
\hline \multicolumn{3}{|l|}{ Index HIV status } \\
\hline Negative & 50 & 98 \\
\hline Positive & 1 & 1.9 \\
\hline \multicolumn{3}{|l|}{ Referred by } \\
\hline Community Health Volunteer (CHV) & 1 & 1.9 \\
\hline Private Sector (PS) & 8 & 16 \\
\hline Self-Referral (SR) & 42 & 82 \\
\hline Outcome of treatment & & \\
\hline
\end{tabular}




\begin{tabular}{|lll|}
\hline & $\mathbf{N}(\mathbf{5 1})$ & $\mathbf{( \% )}$ \\
\hline Cured (C) & 38 & 74 \\
\hline Transferred out (TO) & 3 & 5.9 \\
\hline Treatment completed (TC) & 10 & 20 \\
\hline \#Treatment after loss to follow up, ${ }^{\text {SExtra-pulmonary }}$ TB, \%Pulmonary TB \\
\hline
\end{tabular}

\section{Characteristics of Student Contacts}

A total of 156 contacts with index TB patients (Fig. 1) with median (IQR) age of 23 (20-23) years were evaluated. $80(51 \%)$ were male and $76(49 \%)$ were household contacts. In total, 120 (77\%) of the contacts were off-campus residents and $46(29 \%)$ spent all time with the index TB cases. Cough, fever, weight loss, sweating at night, and swelling at neck/armpits/groin were present amongst 86 (53\%), 60 (39\%), 69 (44\%), $48(31 \%)$, and $27(17 \%)$ of the contacts respectively. Only $3(1.9 \%)$ of the contacts were HIV infected and $9(5.8 \%)$ of the contacts had an underlying medical condition (Table 2). 
Table 2

Demographic and clinical characteristics of contacts

\begin{tabular}{|c|c|c|}
\hline & $N(156)$ & (\%) \\
\hline \multicolumn{3}{|l|}{ Demographic characteristics } \\
\hline Age, median (IQR) years & \multicolumn{2}{|c|}{$23(20-23)$} \\
\hline \multicolumn{3}{|l|}{ Contact type } \\
\hline Household & 76 & 49 \\
\hline Social & 80 & 51 \\
\hline \multicolumn{3}{|l|}{ Gender } \\
\hline Male & 80 & 51 \\
\hline Female & 76 & 49 \\
\hline \multicolumn{3}{|l|}{ Residence } \\
\hline In-Campus (IC) & 36 & 23 \\
\hline Off-Campus (OFC) & 120 & 77 \\
\hline \multicolumn{3}{|c|}{ Time spent in the same room with index case } \\
\hline All time & 46 & 29 \\
\hline Night only & 42 & 27 \\
\hline Day only & 62 & 40 \\
\hline Others & 4 & 3.8 \\
\hline \multicolumn{3}{|l|}{ Clinical Characteristics } \\
\hline Coughing $\geq 2$ weeks & 86 & 53 \\
\hline Fever & 60 & 39 \\
\hline Had weight loss & 69 & 44 \\
\hline Drenching night sweats & 48 & 31 \\
\hline Swelling at neck/armpits/groin & 27 & 17 \\
\hline \multicolumn{3}{|l|}{ HIV test results } \\
\hline Positive & 3 & 1.9 \\
\hline Negative & 70 & 45 \\
\hline Unknown/not tested & 83 & 53 \\
\hline
\end{tabular}




\begin{tabular}{|llc|}
\hline & N (156) & (\%) \\
\hline Any underlying medical condition & 9 & 5.8 \\
\hline
\end{tabular}

\section{Characteristics of Contacts with TB}

Of the 156 contacts with index TB cases, 5 (3.2\% (95\% Cl 1.0 to $7.3 \%)$ tested positive for TB using GeneXpert. The distribution of contact type, age, sex, relation to the index case, and time spent with index case was not different between contacts who contracted TB and those who did not (Fisher's exact Pvalue $>0.05$ ). The distribution of sleeping in the same room with an index case had a borderline difference between contacts with and without TB (Fisher's exact P-value $=0.06$ ). There was evidence of significant distribution of sharing a bed with index case between contacts with and without TB (Fisher's exact $\mathrm{P}=0.003)$ Table 3. 8/156 (5.1\%, (95\% $\mathrm{Cl} 2.2$ to $10 \%)$ contacts, had clinical diagnosed TB despite having a negative GeneXpert result. In total $13 / 156$ contacts had either confirmed or clinically diagnosed TB; $8.3 \%$ (95\% $\mathrm{Cl} 4.5$ to $14 \%$ ). All the 13 TB confirmed and presumptive patients were linked to $\mathrm{KCH}$ to commence treatment. 
Table 3

Comparison of contacts with and without GeneXpert diagnosed TB.

\begin{tabular}{|c|c|c|c|}
\hline & TB $(\mathrm{N}=5)$ & No TB $(N=151)$ & P-value\# \\
\hline \multicolumn{4}{|l|}{ Demographic characteristics } \\
\hline \multicolumn{4}{|l|}{ Contact type $-\mathrm{N}(\%)$} \\
\hline Household & $4(5.3)$ & $72(95)$ & \multirow[t]{2}{*}{0.17} \\
\hline Social & $1(1.3)$ & $79(99)$ & \\
\hline \multicolumn{4}{|l|}{ Age group $-\mathrm{N}(\%)$} \\
\hline$<20$ years & $1(2.0)$ & $49(98)$ & \multirow[t]{3}{*}{0.57} \\
\hline $20-25$ years & $4(4.8)$ & $79(95)$ & \\
\hline$>25$ years & 0 & $23(100)$ & \\
\hline \multicolumn{4}{|l|}{ Gender $-\mathrm{N}(\%)$} \\
\hline Male & $2(2.5)$ & $78(98)$ & \multirow[t]{2}{*}{0.48} \\
\hline Female & $3(4.0)$ & $73(96)$ & \\
\hline \multicolumn{4}{|l|}{ Relation to index case $-\mathrm{N}(\%)$} \\
\hline Relative & $1(2.4)$ & $41(98)$ & \multirow[t]{4}{*}{0.58} \\
\hline Friend & $2(2.5)$ & $78(98)$ & \\
\hline Student & 0 & $7(100)$ & \\
\hline Others & $2(7.4)$ & $25(93)$ & \\
\hline \multicolumn{4}{|l|}{ Time spent with index case $-\mathrm{N}(\%)$} \\
\hline All time & $3(6.5)$ & $43(94)$ & \multirow[t]{4}{*}{0.60} \\
\hline Night only & $1(2.4)$ & $41(98)$ & \\
\hline Day only & $1(1.6)$ & $61(98)$ & \\
\hline Others & 0 & $6(100)$ & \\
\hline Share a bed with index case $-\mathrm{N}(\%)$ & $4(15)$ & $22(85)$ & 0.003 \\
\hline Sleep in same room with index $-\mathrm{N}(\%)$ & $4(7.1)$ & $52(93)$ & 0.06 \\
\hline
\end{tabular}

In the univariate log-binomial regression models, sharing a bed was significantly associated with being TB infected among the contacts. Contact type, age, sex, and time spent with index cases were not associated with being TB infected among the contacts. However, sleeping in the same room with an 
index case had a borderline association with being TB infected among the contacts $(P=0.07)$. In the multivariate regression analysis, only sharing a bed with index case; adjusted risk ratio $21.0(95 \% \mathrm{Cl} 2.45$, 179.8), was associated with being TB infected among the contacts Table 4.

Table 4

Factors associated with contacts being GeneXpert diagnosed TB infected.

\begin{tabular}{|c|c|c|c|c|}
\hline & CRR $(95 \% \mathrm{Cl})$ & P-value & ARR (95\% Cl)\# & P-value \\
\hline \multicolumn{5}{|l|}{ Contact type } \\
\hline Household & Reference & & & \\
\hline Social & $0.24(0.03-2.08)$ & 0.19 & & \\
\hline Age in years & $0.99(0.87-1.12)$ & 0.84 & & \\
\hline \multicolumn{5}{|l|}{ Gender } \\
\hline Male & Reference & & & \\
\hline Female & $1.60(0.26-9.87)$ & 0.61 & & \\
\hline \multicolumn{5}{|c|}{ Time spent with index case } \\
\hline All time & Reference & & & \\
\hline Night only & $0.35(0.03-3.49)$ & 0.37 & & \\
\hline Day only & $0.23(0.02-2.34)$ & 0.21 & & \\
\hline \multicolumn{5}{|c|}{ Share a bed with index case } \\
\hline No & Reference & & Reference & \\
\hline Yes & $20.0(2.33-171.8)$ & 0.006 & $21.0(2.45-179.8)$ & 0.005 \\
\hline \multicolumn{5}{|c|}{ Sleep in same room with index case } \\
\hline No & Reference & & & \\
\hline Yes & $7.62(0.83-68.90)$ & 0.07 & & \\
\hline
\end{tabular}

\section{Discussion}

This pilot study found the collaborative approach was feasible and indicates transmission exists among the student population receiving TB treatment. The study estimated prevalence of $3.2 \%$ is similar to the pooled prevalence of active TB (all forms) among close contacts of $3.1 \%$ and $4.5 \%$ reported in two comprehensive meta-analyses, one of which included 95 contact investigation studies in resource-limited 
countries $[10,17]$. Our estimated prevalence was higher than the reported prevalence of $1.2 \%$ among adults in India [18] and 1.5\% reported in a comparative meta-analysis study in eleven high burden countries [19]. It was however lower than the prevalence of $6 \%$ reported in a Ugandan study [20]. The reported variation in yield of contact investigation studies could be influenced by factors such as background prevalence of TB or HIV infection, design, and implementation of studies which includes screening strategies to identify and trace contacts and diagnostics methods used.

The study observed that sharing a bed with an index case was associated with the risk of contracting TB. Similarly, a study of risk factors for TB among close contacts reported that sharing a bedroom with an index case increased exposure to TB perhaps due to enhanced contact during the night, shared airspace, and sharing of MTB aerosols [21]. Our findings were also consistent with earlier studies where the risk of transmission was reported to be related to the duration and proximity of contact with the source case, being in an enclosed space with the source case and the infectivity of the source case [21-23]. Those who shared a bed likely spent the most time with the patient during their period of infectivity (usually weeks to months before diagnosis) hence at the highest risk of infection. One study showed that symptomatic contacts with diagnosed TB had a comparatively longer duration of symptoms and greater duration of contact with index cases [18]. These findings suggest that the duration of symptoms and duration of contact with index cases could be important predictors for the risk of TB among symptomatic contacts. In the setting of this study students share an average of four continuous months in the same room (duration of one university semester). This cross-sectional study sampled the contacts at a single point within this period, potentially before subsequent transmission from index cases to contacts occurred. It is therefore plausible the transmission rate in this study is a gross underestimation of actual transmission in this population. Future research, including a well-powered prospective cohort study, may improve our understanding of disease transmission.

Understanding the global threat of the youth as an infection pool and therefore potentially transmission to the wider population is of paramount importance in the global response to infectious diseases like TB and COVID 19. Closure of learning institutions and debates on when and how to re-open universities in the absence of a vaccine at the forefront of the COVID 19 strategic plans. Despite the small-scale nature of this pilot study, students played a major role in TB transmission. This timely study contributes to the limited knowledge of contact investigation in the youth population. The resource-limited context of the study demonstrated the feasibility of identifying the youth in universities with airborne infectious diseases and their social contacts. Collaborating and strengthening existing health systems shown in this study promotes identifying youth with TB and facilitates contact tracing. This paper highlights the importance of contact tracing among index cases of infectious disease, similar to TB, among students and justifies further research on the magnitude of transmission of airborne diseases among university students.

\section{Study Strengths and Limitations}

Page $13 / 18$ 
The main strength of this study was the systematic TB testing of all the contacts using GeneXpert which was reported to have high sensitivity [24] to detect TB compared to other diagnostic methods, hence very few TB cases were likely to have been missed. Collaboration with the Kilifi County TB Control programme enabled investigators to work with clinicians who helped in the identification of index patients ensuring that no student TB case was missed. The investigators also worked with Community health volunteers (CHV) who helped navigate the community where students live and aided in the recruitment of index patients and student contacts residing off-campus.

It is possible some students were on TB treatment from other hospitals. Therefore, this study captures a limited proportion of students with TB infection on treatment at $\mathrm{KCH}$ and thus under-represents the magnitude of TB among students. The convenience and operational design of this study using routine hospital data missed a majority of students. The stigma of a TB diagnosis, partly due to the association with HIV in this context, and age group promotes the testing and management of the university population within the vibrant private sector in Kilifi County. This population is mobile and $80 \%$ come from outside the study setting where diagnosis and treatment occur. Inclusion of the private and out of town facilities was not pursued due to time and resource constraints to conduct a larger study.

Currently no TB screening of students, either on admission or at regular intervals during attendance, occurs in Pwani University despite the infection pool they provide. New Students do provide a chest X-ray on admission however the true population with TB infection is unknown.This pilot study underestimates both the magnitude and transmission of TB within the student population.

Lastly, this was an exploratory study and was not powered to test features associated with TB transmission. Furthermore, the investigators did not have the capacity to perform genome sequencing to confirm index case-contacts transmission.

\section{Conclusion and Recommendations}

Students sleeping in crowded hostels promote TB transmission within universities, informing national TB control interventions. Collaborating with existing national TB programme systems is a feasible approach to recruit people with active disease and their social contacts. Expansion of this approach to a larger population of students with TB infection may demonstrate the magnitude of TB transmission within universities and their wider communities.

\section{Declarations}

\section{Declarations}

\section{Ethics approval and consent to participate}


Ethical approval was obtained from the Pwani University Ethical Review Committee (ERC/MSc/002/2017). Clearance to obtain information about the students with TB infection was also obtained from the Kilifi County Hospital administration. Informed consent was obtained from the students before they were enrolled into the study.

\section{Consent for publication}

Not applicable.

\section{Availability of data and materials}

The study data are available from the corresponding author on reasonable request.

\section{Competing interests}

The authors declare that they have no competing interests.

\section{Funding}

This study was funded by the Pwani University Internal Research Grant. The funder had no role in study design, data collection, analysis, interpretation and writing of the manuscript.

\section{Authors' contributions}

MT, OA and WA conceived the idea. MT recruited study participants and coordinated the study operation. NM developed the study database and conducted statistical analysis. MT drafted the first manuscript which was further developed, reviewed, and approved by all authors.

\section{Acknowledgements}

Authors would like to acknowledge participants, Kilifi County Hospital, and Community Health Volunteers for making this study a success.

\section{References}

1. WHO. Global tuberculosis report 2016. Geneva; 2016.

https://apps.who.int/iris/handle/10665/250441. 
2. WHO. Global tuberculosis report 2018. Geneva; 2018. https://apps.who.int/iris/handle/10665/274453.

3. Snow KJ, Nelson LJ, Sismanidis C, Sawyer SM, Graham SM. Incidence and prevalence of bacteriologically confirmed pulmonary tuberculosis among adolescents and young adults: a systematic review. Epidemiol Infect. 2018;146:946-53. doi:10.1017/S0950268818000821.

4. Storla DG, Yimer S, Bjune GA. A systematic review of delay in the diagnosis and treatment of tuberculosis. BMC Public Health. 2008;8:15. doi:10.1186/1471-2458-8-15.

5. Finnie RKC, Khoza LB, van den Borne B, Mabunda T, Abotchie P, Mullen PD. Factors associated with patient and health care system delay in diagnosis and treatment for TB in sub-Saharan African countries with high burdens of TB and HIV. Trop Med Int Heal. 2011;16:394-411. doi:10.1111/j.13653156.2010.02718.x.

6. Sullivan BJ, Esmaili BE, Cunningham CK. Barriers to initiating tuberculosis treatment in sub-Saharan Africa: a systematic review focused on children and youth. Glob Health Action. 2017;10:1290317. doi:10.1080/16549716.2017.1290317.

7. WHO. Recommendations for investigating contacts of persons with infectious tuberculosis in lowand middle-income countries. Geneva; 2012. doi:WHO/HTM/TB/2012.9.

8. González-Ochoa E, Brooks JL, Matthys F, Calisté P, Armas L, Van der Stuyft P. Pulmonary tuberculosis case detection through fortuitous cough screening during home visits. Trop Med Int Heal. 2009;14:131-5. doi:10.1111/j.1365-3156.2008.02201.x.

9. Fox GJ, Nhung NV, Sy DN, Hoa NLP, Anh LTN, Anh NT, et al. Household-Contact Investigation for Detection of Tuberculosis in Vietnam. N Engl J Med. 2018;378:221-9. doi:10.1056/NEJMoa1700209.

10. Fox GJ, Barry SE, Britton WJ, Marks GB. Contact investigation for tuberculosis: a systematic review and meta-analysis. Eur Respir J. 2013;41:140-56. doi:10.1183/09031936.00070812.

11. Sandgren A van der. WM. ECDC guidance on management of contacts of MDR-TB and XDR-TB patients. Eur Respir J. 2012. https://erj.ersjournals.com/.

12. Marais BJ, Lönnroth K, Lawn SD, Migliori GB, Mwaba P, Glaziou P, et al. Tuberculosis comorbidity with communicable and non-communicable diseases: integrating health services and control efforts. Lancet Infect Dis. 2013;13:436-48. doi:10.1016/S1473-3099(13)70015-X.

13. Faccini M, Codecasa LR, Ciconali G, Cammarata S, Borriello CR, De Gioia C, et al. Tuberculosis Outbreak in a Primary School, Milan, Italy. Emerg Infect Dis. 2013;19:485-7. doi:10.3201/eid1902.120527.

14. Mekonnen A, Petros B. Burden of tuberculosis among students in two Ethiopian universities. Ethiop Med J. 2016. https://www.emjema.org/index.php/EMJ.

15. Enos M, Sitienei J, Ong'ang'o J, Mungai B, Kamene M, Wambugu J, et al. Kenya tuberculosis prevalence survey 2016: Challenges and opportunities of ending TB in Kenya. PLoS One. 2018;13:e0209098. doi:10.1371/journal.pone.0209098. 
16. Abdullahi OA, Ngari MM, Sanga D, Katana G, Willetts A. Mortality during treatment for tuberculosis; a review of surveillance data in a rural county in Kenya. PLoS One. 2019;14:e0219191. doi:10.1371/journal.pone.0219191.

17. Morrison J, Pai M, Hopewell PC. Tuberculosis and latent tuberculosis infection in close contacts of people with pulmonary tuberculosis in low-income and middle-income countries: a systematic review and meta-analysis. Lancet Infect Dis. 2008;8:359-68. doi:10.1016/S1473-3099(08)70071-9.

18. Gupta M, Saibannavar A, Kumar V. Household symptomatic contact screening of newly diagnosed sputum smears positive tuberculosis patients - An effective case detection tool. Lung India. 2016;33:159. doi:10.4103/0970-2113.177445.

19. Blok L, Sahu S, Creswell J, Alba S, Stevens R, Bakker MI. Comparative Meta-Analysis of Tuberculosis Contact Investigation Interventions in Eleven High Burden Countries. PLoS One. 2015;10:e0119822. doi:10.1371/journal.pone.0119822.

20. Guwatudde D. Tuberculosis in Household Contacts of Infectious Cases in Kampala, Uganda. Am J Epidemiol. 2003;158:887-98. doi:10.1093/aje/kwg227.

21. Reichler MR, Khan A, Sterling TR, Zhao H, Chen B, Yuan Y, et al. Risk Factors for Tuberculosis and Effect of Preventive Therapy Among Close Contacts of Persons With Infectious Tuberculosis. Clin Infect Dis. 2020;70:1562-72. doi:10.1093/cid/ciz438.

22. Fok A, Numata Y, Schulzer M, FitzGerald MJ. Risk factors for clustering of tuberculosis cases: $A$ systematic review of population-based molecular epidemiology studies. International Journal of Tuberculosis and Lung Disease. 2008. https://www.theunion.org/what-we-do/journals/ijtld.

23. Greenaway C, Palayew M, Menzies D. Yield of casual contact investigation by the hour. Int J Tuberc Lung Dis. 2003. https://www.theunion.org/what-we-do/journals/ijtld.

24. WHO. Xpert MTB/RIF implementation manual: technical and operational 'how-to'; practical considerations. Geneva; 2014. https://apps.who.int/iris/handle/10665/112469.

\section{Figures}




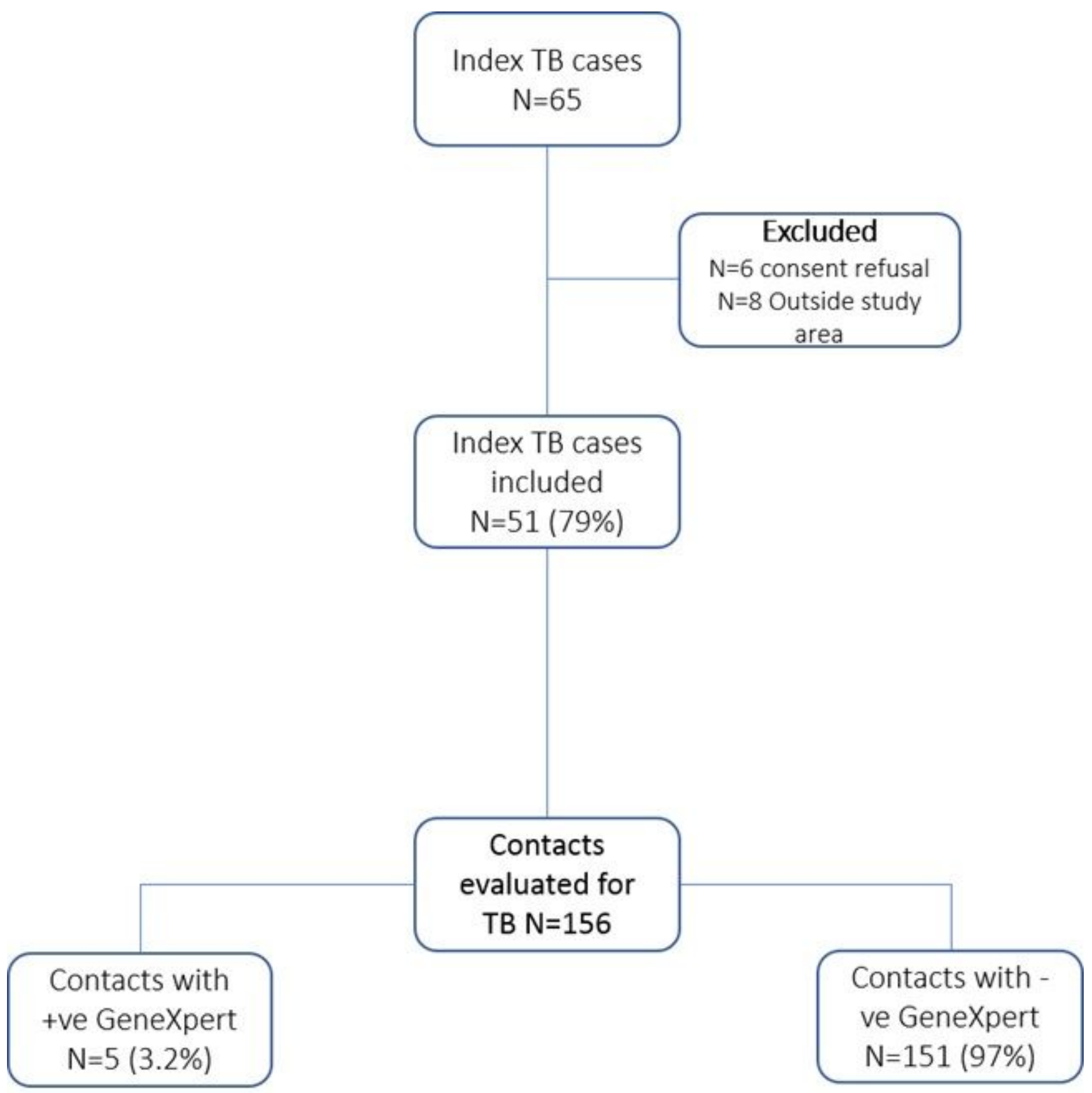

Figure 1

Flow chart of the study participants 